\title{
Immunohistochemical and Electron Microscopic Study of the Biodegradation Processes of Chitin and Chitosan Implanted in Rat Alveolar Bone
}

\author{
Takeshi Ikeda, Kajiro Yanagiguchi, Tunenori Mastunaga, Shiduka Yamada, Naoko Ohara, Tomoko Ganno, \\ and Yoshihiko Hayashi \\ Division of Cariology, Department of Developmental and Reconstructive Medicine, Course of Medical and Dental Sciences Nagasaki \\ University Graduate School of Biomedical Sciences, Nagasaki, Japan
}

\begin{abstract}
Ikeda T, Yanagiguchi K, Mastunaga T, Yamada S, Ohara N, Ganno T and Hayashi Y. Immunohistochemical and electron microscopic study of the biodegradation processes of chitin and chitosan implanted in rat alveolar bone. Oral Med Pathol 2005; 10: 131-138, ISSN 1342-0984
\end{abstract}

The present study was designed to investigate histochemically the biodegradation processes of chitin and chitosan implanted in rat alveolar bone. Lysozyme was immunohistochemically detected using postembedding immunogold labeling. The degradation process was ultrastructurally observed using the lectin-colloidal gold technique with electron microscopy. Three groups of chitin were specially prepared according to their degree of deacetylation: 100\% deacetylated chitin (DDAC 100); 50\% (DDAC 50); and 0\% (DDAC 0). The present immunohistochemical study indicated that lysozyme expression was not detected in the DDAC 100 group. Furthermore, electron microscopy clearly demonstrated that the contour of implanted chitosan changed over time, and that chitosan-like fragments were present in the phagosomes in the DDAC 50 and 100 groups. These findings strongly suggest that phagocytes, such as multinuclear cells, are easily supplied in bone tissue and that the phagocytosis is more effective than enzymatic digestion for chitin and chitosan biodegradation in bone tissue. DDAC 100 should be a suitable biomaterial for bone surgery and bone regeneration therapy.

Key words: chitosan, lysozyme, phagocytosis, biodegradation, bone healing

Correspondence: Takeshi Ikeda, Division of Cariology, Department of Developmental and Reconstructive Medicine, Course of Medical and Dental Sciences Nagasaki University Graduate School of Biomedical Sciences, 1-7-1 Sakamoto, Nagasaki, 852-8588, Japan

Phone: +81-95-849-7679, Fax: +81-95-849-7680

\section{Introduction}

Chitin exists in the exoskeleton of crustaceans, such as insects, crabs and shrimps and in the cell wall of the bacillus $(1,2)$. It is known that chitosan, obtained by the alkaline deacetylation of chitin in industrial processing (3), posseses excellent biological properties for accelerating wound healing (4-6) and enhancing osteogenesis (7). Furthermore, the biodegradation of chitosan through hydrolytic enzymes (mainly lysozyme) can release aminosugars that are incorporated into glycosaminoglycans and glycoproteins (8-10). In addition, chitosan as a biocompatible $(11,12)$ and bioactive polymer stimulates the immune system of the host against viral and bacterial infections by activating macrophages (13-16). These bioactive characteristics have led to its application as a regeneration material for hard and soft tissue, and as an absorbable suture (17), with many uses, such as in films, sponges, fibers, solutions, powders, flakes and gels.
In dentistry, chitin and its derivatives have also been applied as a dressing for oral mucosal wounds and as a tampon following radical treatment of maxillary sinusitis (18). Previously, we showed that when a refractory-infected root canal having a perpetuating exudate was treated, the cotton-like chitin was useful for controling inflammatory exudates. In addition, the use of chitin decreased the clinical symptoms and signs, coinciding with a reduction of the lysozyme concentration in the periapical regions over time through the use of intracanal medication (19).

Chitin and its derivatives play a role in hyperplasia of granulation tissue and the vascular system, finally inducing osteoblast-like cell proliferation. Consequently, bone defects are filled with osseous tissue (20, 21). However, little is known about how chitin and chitosan are degraded in bone tissue. Therefore, the present study was designed to investigate histochemically the biodegrada- 
tion processes of implants in rat alveolar bone defects.

\section{Material and Methods}

Animal experiments

All animal experiments were conducted in accordance with the Guidelines for Animal Experiments, Nagasaki University, and as permitted by the Committee of Biomedical Research Center for Laboratory Animals, Center for Frontier Life Sciences, Nagasaki University.

Sixty Wistar male rats weighing about $160 \mathrm{~g}$ were used for this experiment. Chitin and its derivatives were kindly supplied by UNITIKA Co., Ltd. (Tokyo, Japan). The degrees of deacetylation of the specially- prepared chitin were 0,50 and $100 \%$. We refer to these as degree of deacetylated chitin (DDAC) 0, DDAC 50 and DDAC 100, respectively. Each group consisted of 20 rats and was divided into four postoperative groups, representing the postoperative time periods of weeks $1,2,3$ and 4 . Anesthesia was performed using an intraperitoneal injection of sodium pentobarbital $(25.9 \mathrm{mg} / \mathrm{kg})$. A skin incision was performed along the inferior border of the mandible. The periosteal flap was deflected, and two cylindrical cavities were prepared below the external oblique protuberance (near the posteroinferior portion of the mental foramen) on both sides of the mandible. During the entire procedure, a sterile \# 1 round bur was used at a low speed. The cavity size was about $1 \mathrm{~mm}$ in both diameter and depth. Complete hemostasis inside the prepared cylindrical cavities was accomplished by irrigation with physiological saline. Three kinds of different degrees of cotton-like deacetylated chitin, weighing about $0.5 \mathrm{mg}$, were implanted in the each of the three cavities without sealing, respectively. A fourth cavity without chitin was used as a control. The periosteal flap was finally repositioned and sutured.

\section{Preparation of specimens}

Rats were perfused through the left ventricle with the fixative containing $4 \%$ paraformaldehyde, $0.1 \%$ glutaraldehyde, $0.2 \%$ picric acid and $4 \%$ polyvinylpyrrolidone (PVP) in $0.1 \mathrm{M}$ phosphate buffer ( $\mathrm{pH}$ 7.2) under deep sodium pentobarbital anesthesia. The implanted chitosan, together with the surrounding bone, was carefully dissected with a diamond disk under a stream of Locke's solution. The blocks were dehydrated in increasing concentrations of methanol and then embedded in LR gold (London Resin, Basingstoke, UK). The UV initiator, benzoin methyl ether was applied at a concentration of $0.5 \%$ w/v. The LR gold/initiator mixture was gently stirred to minimize the introduction of oxygen, which inhibited polymerization. The blocks were transferred into gelatin capsules (size 0 ) and placed at $4^{\circ} \mathrm{C}$. Every capsule was tightly sealed to exclude atmospheric oxygen during polymerization. The capsules were placed in ultraviolet polymerizer (TUV-100, Dosaka, Osaka, Japan) at $-25^{\circ} \mathrm{C}$ for $24 \mathrm{~h}$.

Immunohistochemistry of implanted chitin and chitosan Each group of undemineralized specimens was cut with glass knives. Semithin sections (about $1 \mu \mathrm{m}$ ) were stained with toluidine blue for light microscopic orientation. For the immunohistochemical study, semithin sections were preincubated with a blocking solution, consisting of $1 \%$ immunoglobulin-free bovine serum albumin (BSA) (Fraction V, Sigma Chemical, St Louis, Mo., USA) and 5\% normal goat serum (Sigma Chemical) in $0.05 \mathrm{M}$ phosphate buffer saline (PBS) (pH 7.4) for $30 \mathrm{~min}$ at room temperature (RT). The sections were washed with an incubation buffer, composed of $0.2 \%$ acetylated BSA (Aurion, Wageningen, Netherlands), 0.01\% Tween 20 (Sigma Chemical), and $0.03 \%$ Triton X-100 (Serva, Heidelberg, Germany) in $0.05 \mathrm{M}$ PBS for $5 \mathrm{~min}$ at RT, followed by incubation with rabbit polyclonal anti-human lysozyme antibody (Dako Cytomation, Glostrup, Denmark) for $20 \mathrm{~h}$ at $4^{\circ} \mathrm{C}$ in a relative humidity of $100 \%$. After rinsing with incubation buffer for 5 min ( 6 times), the tissue sections were incubated with a 12-nm colloidal gold-AffiniPure goat anti-rabbit IgG (EM Grade, Jackson Immunoresearch Lab, West Grove, Pa., USA) for $2 \mathrm{~h}$ (22). For the visualization of lysozyme at the light microscopic level, the site of immunogold labeling was developed with a silver-enhancing kit (British BioCell International, UK) at $\mathrm{RT}$ for $10 \mathrm{~min}$, followed by counterstaining with nuclear fast red for $5 \mathrm{~min}$.

For immunohistochemical controls, sections were incubated directly with the IgG-gold complex without the primary antibody and were also incubated with an antilysozyme antibody adsorbed with sufficiently purified lysozyme (hen egg white, Sigma Chemical) for $24 \mathrm{~h}$ at 4 ${ }^{\circ} \mathrm{C}$. Moreover, sections were incubated with a normal rabbit immunoglobulin fraction (X, Dako Cytomation) diluted to the same extent as the specific antibody (1:100).

\section{Electron microscopy of implanted chitin and chitosan}

Colloidal gold was prepared by reducing chloroauric acid $\left(\mathrm{HAuCl}_{4} \cdot 4 \mathrm{H}_{2} \mathrm{O}\right.$, Nakalai Tesque, Kyoto, Japan) with trisodium citrate. In this research, particles $11 \mathrm{~nm}$ in diameter were used and the specific lectin for N-acetyl-Dglucosamine, wheat germ agglutinin (WGA, EY Laboratories, San Mateo, CA, USA), was conjugated with colloidal gold $(23,24)$.

For labeling with WGA-gold, ultrathin sections mounted onto nickel grids were incubated with $1 \%$ bovine serum albumin in $0.01 \mathrm{M}$ PBS for $5 \mathrm{~min}$ and then transferred to WGA-gold conjugates $(50 \mu \mathrm{g} / \mathrm{ml})$ and incubated for $30 \mathrm{~min}$ at RT. The sections were washed with PBS, rinsed with distilled water, and dried. After brief staining with uranyl acetate and lead citrate, sections were examined using a Hitachi H-7100 transmission elec- 

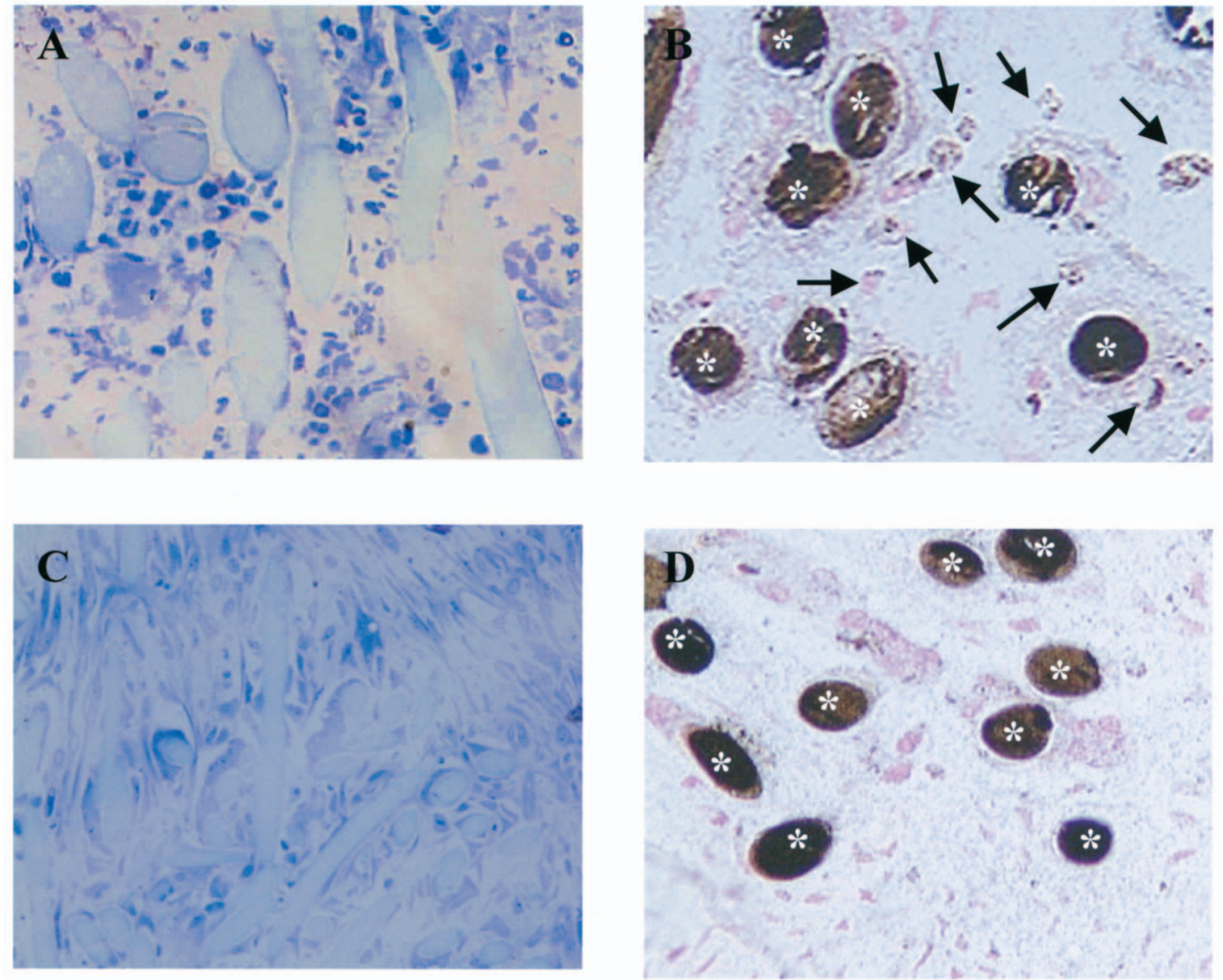

Fig. 1: Light micrographs in the DDAC 0 group.

(A) Polymorphonuclear leukocytes are surrounding chitin fibers at 1 week (toluidine blue staining, original magnification; $\times 400$ ).

(B) Lysozyme positive cells (arrows) are accumulated around chitin fibers $(*)$ at 1 week (immunogold-silver staining, original magnification; $\times 600$ ).

(C) Chitin fibers are encaspulated with the granulation tissue at 4 weeks (toluidine blue staining, original magnification; $\times 400$ ).

(D) Lysozyme positive cells are not visible at 4 weeks (immunogold-silver staining, original magnification; $\times$ 600). * : chitin fibers.

tron microscope to observe the localization of chitin remnants in the bone tissue. For controls, the specificity of lectin binding was tested by the addition of $0.1 \mathrm{M}$ of $\mathrm{N}-$ acetyl-D-glucosamine (Sigma Chemical) as the appropriate inhibitory sugar to WGA or WGA-gold complexes 30 min before incubation.

\section{Results}

Immunohistochemistry of implanted chitin and chitosan The histopothological examination revealed different tissue reactions depending on degree of deacetylation from 1 to 4 weeks postoperatively.

In the case of DDAC 0 , at 1 week many neutrophils were accumulated around chitin fibers and a relatively severe inflammatory reaction occurred (Fig. 1A). Immunogold-silver staining for lysozyme showed that accumulated neutrophils were positive for lysozyme and that very intensive lysozyme expression on the chitin fibers themselves was also visible. As a substrate for the lysozyme, chitin fibers were strongly stained brownishblack with anti-lysozyme (Fig. 1B). At 4 weeks, the granulation tissue around the fibers consisted of macrophages and multinuclear giant cells (Fig. 1C). Lysozyme positive cells were not immunohistochemically demonstrated surrounding the fibers (Fig. 1D).

In the case of DDAC 50, acute inflammatory cell infiltration was not observed at 1 week, however, a few mononuclear phagocytes, such as monocyte and macrophage, appeared adjacent to fibers. Some of multinuclear giant cells and newly formed capillaries were also present (Fig. 2A). The immunogold-silver staining showed only a few of the lysozyme positive cells (Fig. 2B). At 2 weeks the immunohistochemical finding showed no lysozyme expressing cells (Fig. 2C). At 3 weeks multinuclear giant cells around the fibers were increased compared with those at 2 weeks, and the encapsulation by fibrous tissue could be recognized (Fig. 2D).

In the case of DDAC 100, at 1 week the monocyte, 

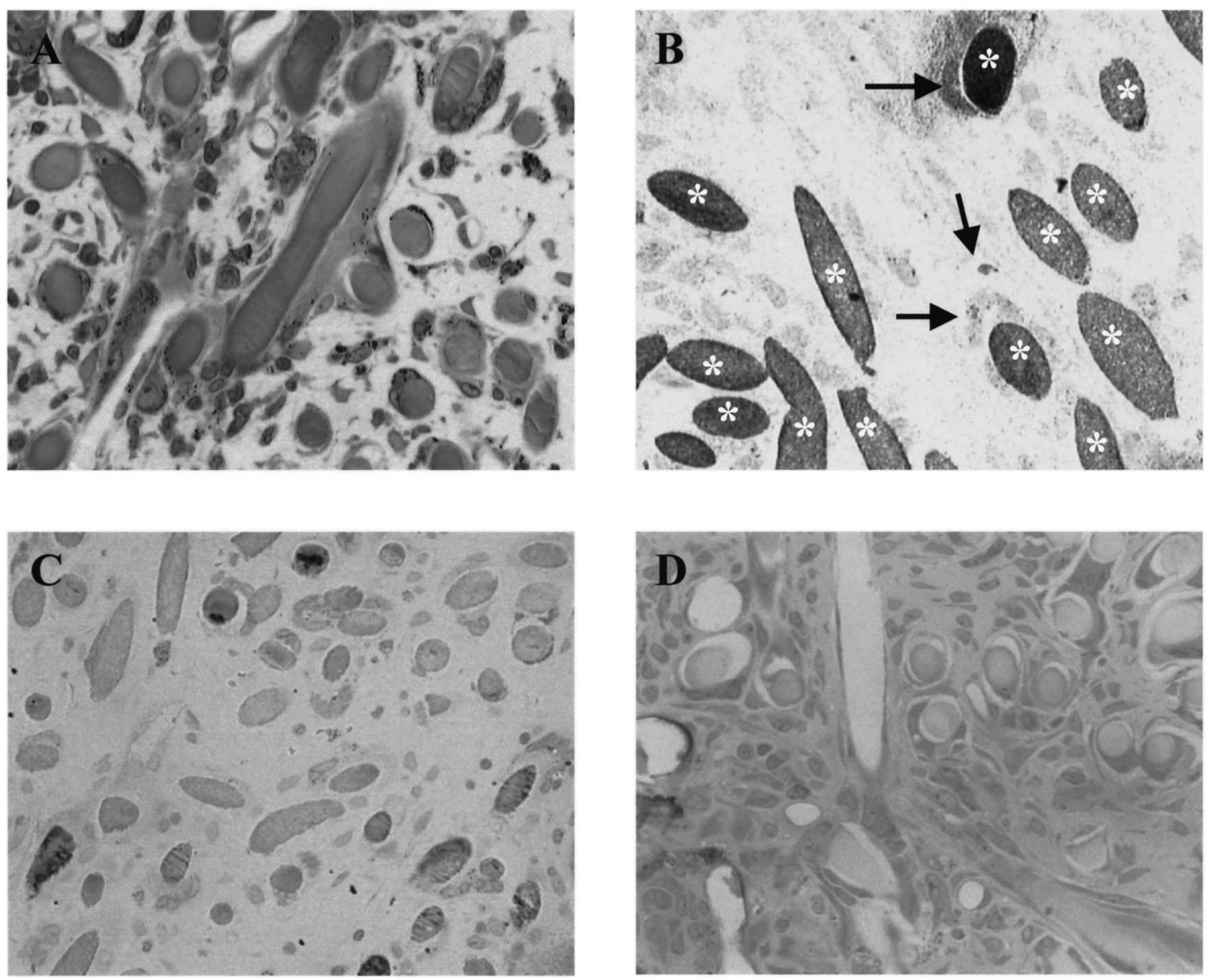

Fig. 2: Light micrographs in the DDAC 50 group.

(A) A few mononuclear phagocytes are adjacent to fibers at 1 week (toluidine blue staining, original magnification; $\times 400$ ).

(B) A few lysozyme positive cells (arrows) are visible around chitin fibers (*) at 1 week (immunogold-silver staining, original magnification; $\times 600)$.

(C) There are no lysozyme positive cells at 2 weeks (immunogold-silver staining, original magnification; $\times 400$ ).

(D) Multinuclear giant cells around the fibers are increased and chitin fibers are encapsulated by fibrous tissue at 3 weeks (toluidine blue staining, original magnification; $\times 400$ ).

macrophage and multinuclear giant cells were moderately infiltrated and the spaces of capillaries and perivascular tissue increased compared with those in DDAC 50 (Fig. 3A). Lysozyme expression was never detected during the 4 weeks of the experimental period (Fig. 3B). At 2 weeks multinuclear giant cells showed greater accumulation around the fibers in DDAC 100 than in DDAC 50. Many newly-formed capillaries were observed in the granulation tissue (Fig. 3C).

\section{Electron microscopy of implanted chitin and chitosan}

TEM examination revealed labeling with WGA-gold particles, indicating the existence of $\mathrm{N}$-acetyl-D-glucosamine. It was clearly evident that the higher the DDA, the weaker the intensity of gold labeling in observed crosssectional fibers.

In the case of DDAC 0, at 1 week postoperatively, neutrophils containing numerous lysosomes were present immediately adjacent to the gold-labeled chitin fibers (Fig. 4). At both 2 and 3 weeks, the electron microscopic findings, such as the accumulation of macrophages and multinuclear giant cells, were observed immediately adjacent to the fibers. At 4 weeks, it was revealed that fibroblasts encapsulated chitin fibers with collagen fibers. However, the surface of the fibers was smooth during all observed periods (Fig. 5).

In the case of DDAC 50, the contour of fibers was not as smooth as in DDAC 0. Both 3 and 4 weeks postoperatively, phagosomes, some of which were gold-labeled (Fig. 6), adhered to the fibers.

In the case of DDAC 100, the contour of chitosan fiber was more irregular than that in the DDAC 50 group (Fig. 7). Multinuclear giant cells with phagosomes containing debris-like structures were observed until 3 weeks (Fig. 8). At 4 weeks, the chitosan fibers were encapsulated by collagen fiber. 

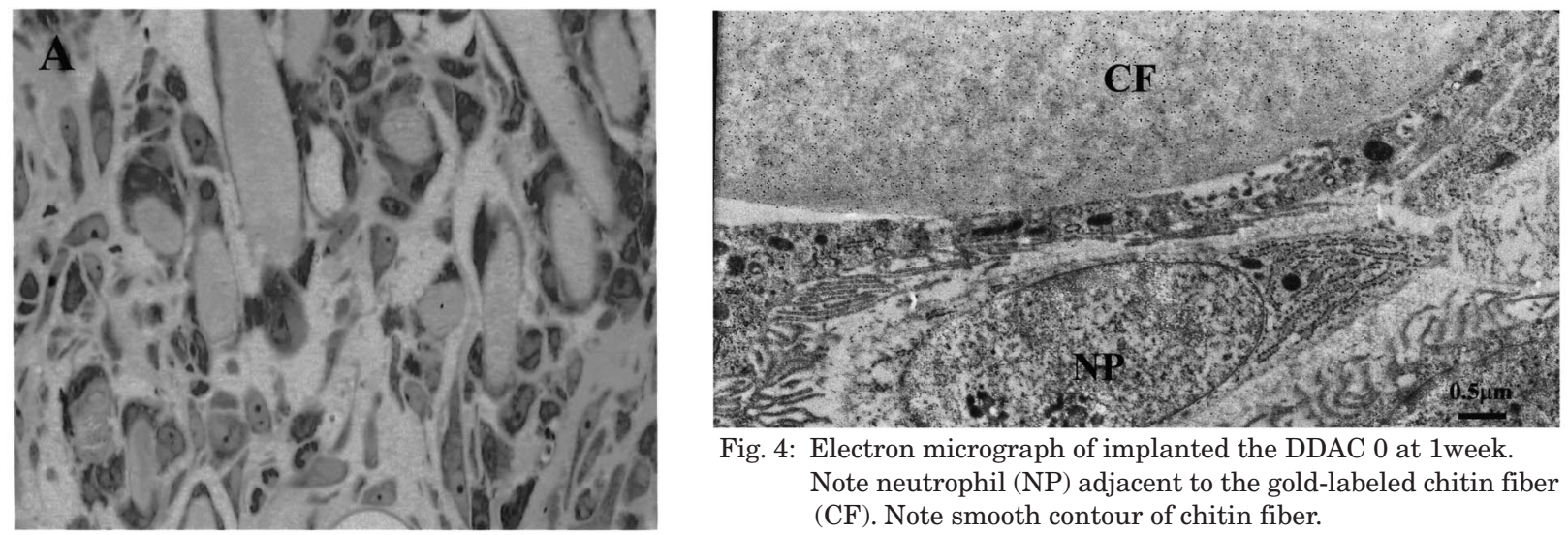

Fig. 4: Electron micrograph of implanted the DDAC 0 at 1 week. Note neutrophil (NP) adjacent to the gold-labeled chitin fiber (CF). Note smooth contour of chitin fiber.
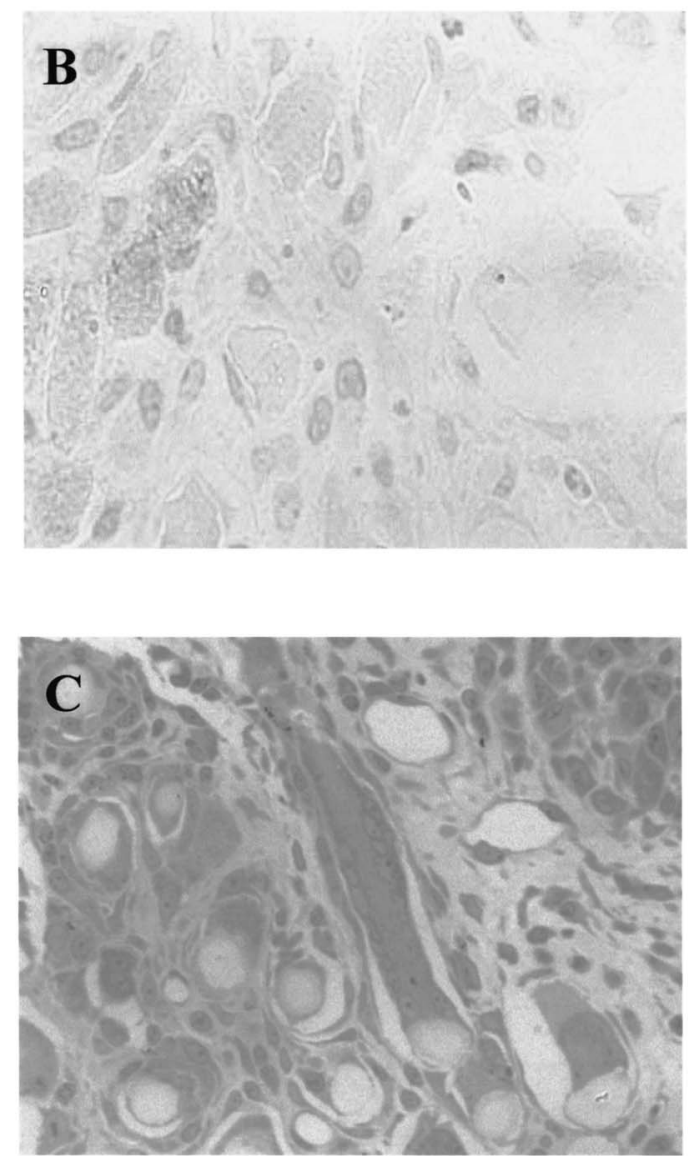

Fig. 3: Light micrographs in the DDAC 100 group.

(A) Monocyte and macrophage are moderately infiltrated at 1 week (toluidine blue staining, original magnification; $\times 400)$.

(B) There are no lysozyme positive cells at 1 week (immunogold-silver staining,original magnification; $\times 400)$.

(C) Numerous multinuclear giant cells around the fibers and capillaries in the granulation tissue are recognized at 2 weeks (toluidine blue staining, originalmagnification; $\times 400$ ).

\section{Discussion}

Chitosan is easily hydrolyzed by various chitosanases $(25,26)$, which are completely absent in mammals, and the hydrolization depends on the degree

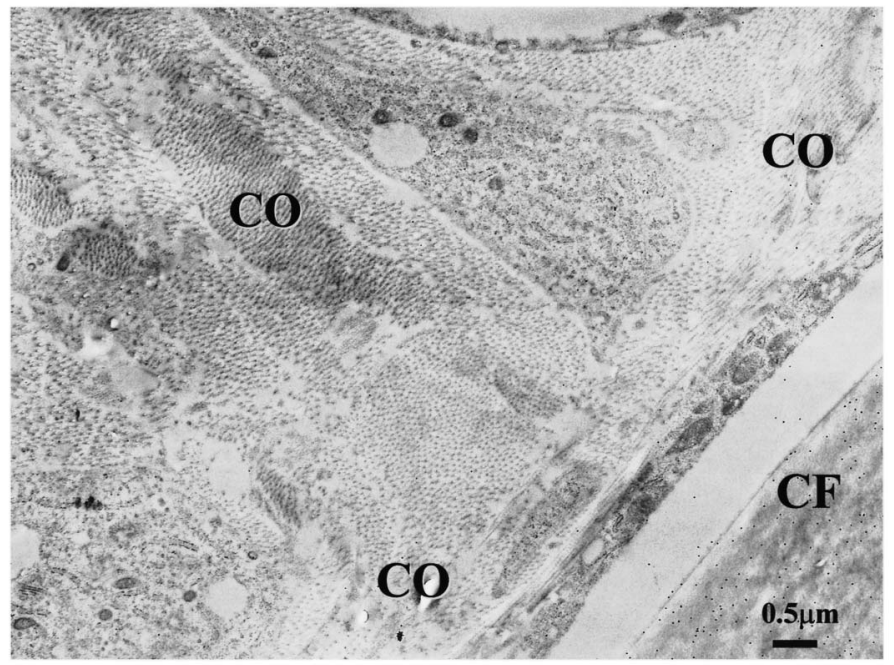

Fig. 5: Electron micrograph of implanted the DDAC 0 at 4 weeks. Note numerous collagen fibers $(\mathrm{CO})$ in the extracellular space. $\mathrm{CF}$ : gold-labeled chitin fiber.

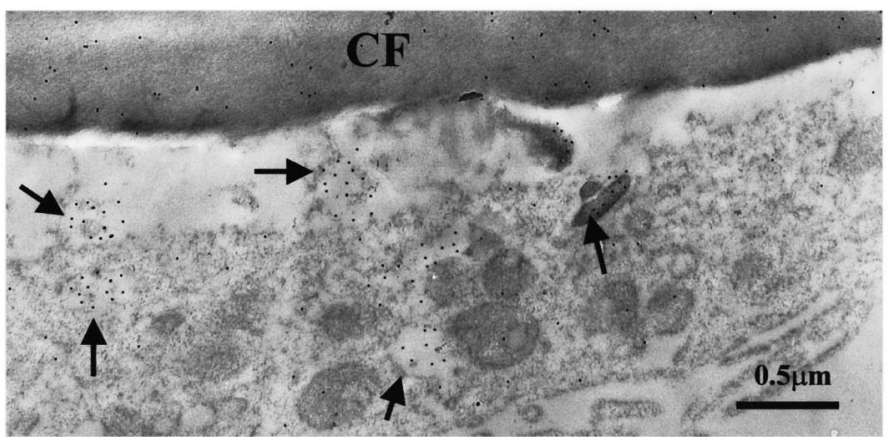

Fig. 6: Electron micrograph of implanted the DDAC 50 at 3 weeks. The multinuclear giant cell with gold-labeled phagosomes (arrows) is adjacent to chitin fiber $(\mathrm{CF})$.

of deacetylation $(27,28)$. Lysozyme, normally produced by macrophages and neutrophils, hydrolyses susceptible modified chitosan to oligomers, which activate macrophages to produce nitric oxide, activated oxygen species, tumor necrosis factor- $\alpha$, interferon and interleukin-1 (16). The biodegradation of chitosan leads to the release of monomeric aminosugars, which can be incorporated into glycosaminoglycan and glycoprotein metabolic pathways, 

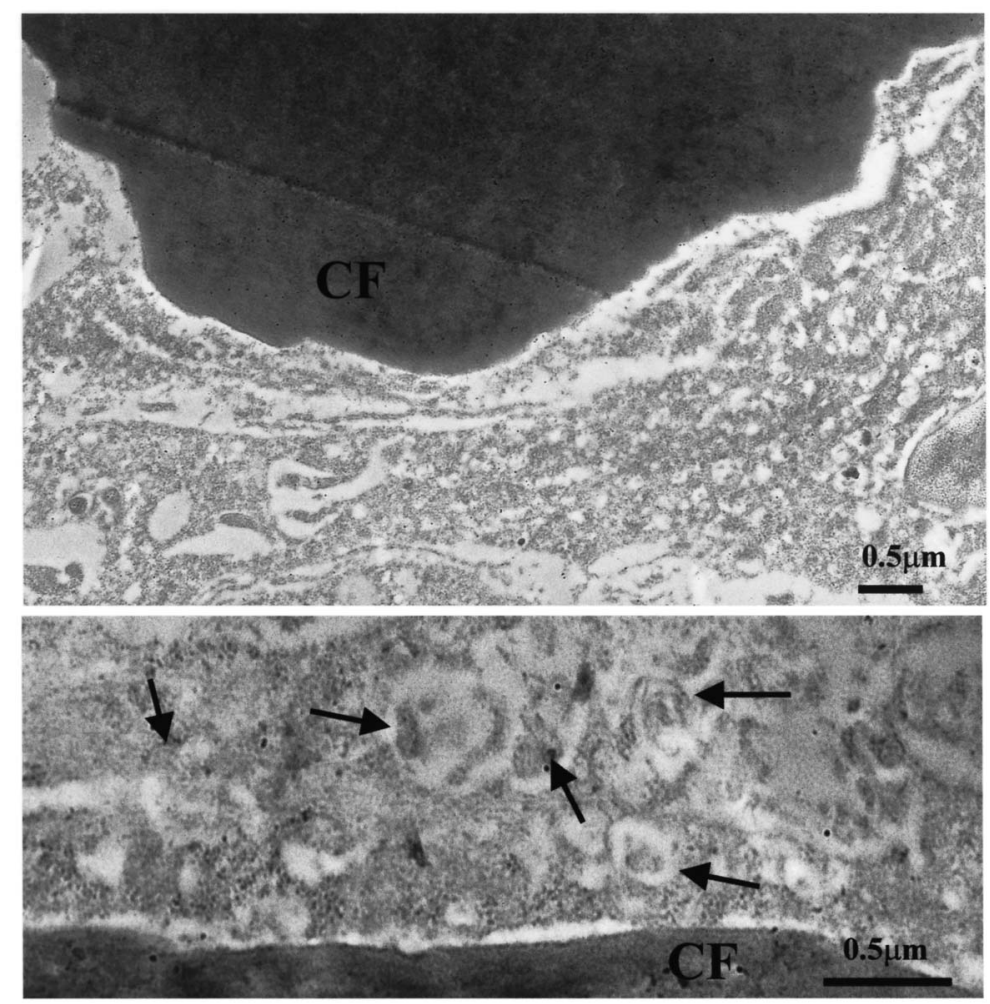

Fig. 7: Electron micrograph of implanted the DDAC 100 at 1 week.

Note the irregular contour of chitosan fiber (CF). thus guiding the ordered deposition of collagen, which is also influenced by chitosan oligomer. The present immunohistochemical study indicated that lysozyme expression was not detected in the DDAC 100 group after 1 week, when most inflammatory cell infiltration had already disappeared. Furthermore, the chitosan monomer (D-glucosamine) might be produced by the intracellular digestion of phagocytosed chitosan, because chitosan has an excellent bioactivity in bone healing (29).

Tissue responses to chitin and its derivatives have been widely studied (30-32). A recent study clearly demonstrated that when implanting the films in the subcutaneous tissue of rats for different periods of time (12), there was an increasing degree of acetylation (DA) of chitosan and decreasing cell adhesion on the film. Many other studies also support this result. DA plays a key role in cell adhesion and proliferation. We have already reported the physiological degradation process of chitosan in bone (33). Cotton-like chitosan (35, 70 and $100 \%$ deacetylation) was implanted into alveolar bone cavities, similarly to the present study. Histopathological examination was carried out after 1, 3, 6, 9 and 12 months of implantation. All types of chitosan were degraded in time, together with bone formation. Especially, in the case of $100 \%$ deacetylated chitin, the cavity was almost completely filled with newly- formed bone tissue after 9 months. These results show that chitosan may promote wound healing in bone tissue as well as in soft tissue.

In vitro degradation of films of chitin and its deacetylated derivatives were examined by immersing the films in buffered aqueous solution of $\mathrm{pH} 7$ containing lysozyme at $37^{\circ} \mathrm{C}(34)$. The in vitro degradation of chitin with $4 \mathrm{mg} / \mathrm{ml}$ lysozyme readily undergoes degradation. In contrast to chitin, deacetylated derivatives with deacetylation higher than $73 \mathrm{~mol} \%$ are virtually resistant against the enzymatic hydrolysis in vitro. This means that DDAC 100 groups were never attacked by the enzymatic hydration. The data is completely consistent with the present immunohistochemical findings.

The in vivo biodegradation was examined by subcutaneously implanting the films in the back of rats (34). It was found that the rate of in vivo biodegradation was very high for chitin and $68.8 \mathrm{~mol} \%$ deacetylated chitin, compared with that for the $73.3 \mathrm{~mol} \%$ deacetylated chitin. The films (more than $73.3 \mathrm{~mol} \%$ deacetylated derivatives) showed slower biodegradation in soft tissue. Our previous (33) and present studies have indicated that the higher the DDAC, the more rapid the biodegradation occurs. The implanted chitosan (DDAC 100) almost disappeared after 6 months postoperatively. This discrepancy was that the former was in the subcutaneous tissue while the latter was in bone. Furthermore, the present electron microscopy clearly demonstrated that the contour of implanted chitosan was changed such that it became irregular, and that phagosomes were observed in the DDAC 50 and 100 groups. These findings strongly suggest that phagocytes, such as multinuclear cells, are easily supplied in bone tissue. These results also indicate that phagocytosis is more effective than enzymatic digestion for chitin and chitosan biodegradation in bone tissue.

Chitin and chitosan have strong chemotactic activity, thus the initially severe inflammatory reaction 
associated with these materials needs to be controlled before clinical application. In the study of deacetylated chitin derivatives implanted subperiosteally over the calvaria, membranes made of $94 \%$ deacetylated chitin and chitosan were reported to show mild inflammation and minimum osteogenesis (35). The difference compared with the present finding is the site of application. As already mentioned, phagocytes could easily be supplied inside the bone tissue and around the implanted chitosan. The molecular weight of chitosan used in this study was about 200,000 DA, and was a polymer type. Furthermore, the present histopathological findings showed that neutrophils were observed only in the chitin group 1 week after implantation. In the DDAC 50 and 100 groups, neutrophils were not observed after 1 week. These results, together with our previous data (33), indicate that DDAC 100 should be a suitable biomaterial for bone surgery and bone regeneration therapy.

\section{Acknowledgments}

This study was supported in part by Grant-in-Aid for Scientific Research 15592023 from the Ministry of Education, Science, Sports and Culture, Japan.

\section{References}

1. Giraudguille MM. Fine-structure of the chitin protein system in the crab cuticle. Tissue Cell 1984; 16: 75-92.

2. Muzzarelli RA. Chitosan In Natural Chelating Polymers. Muzzarelli R. ed. Pergamon Press, Italy. 1973; 144-76.

3. Borzacchiello A, Ambrosio L, Netti PA, et al. Chitosan-baced hydrogels: synthesis and characterization. J Mater Sci Mater Med 2001; 12: 861-4.

4. Biagini G, Muzzarelli RA, Giardino R, et al. Biological materials for wound healing. In: Brine CJ, Sandford A, Zikakis P. ed. Advances in Chitin and Chitosan vol. 1, Elsevier Science, Barking. 1992; 16-24.

5. Mi FL, Shyu SS, Wu YB, et al. Fabrication and characterization of a sponge-like asymmetric chitosan membrane as a wound dressing. Biomaterials 2001; 22: 165-73.

6. Ueno H, Mori T and Fujinaga T. Topical formulations and wound healing applications of chitosan. Adv Drug Deliv Rev 2001; 52: 105-15.

7. Klokkevold PR, Vandemark L, Kenney EB, et al. Osteogenesis enhanced by chitosan (poly- $N$-acetyl glucosaminoglycan) in vitro. $J$ Peridont 1996; 67: 1170-5.

8. Muzzarelli RA. Human enzymatic activities related to the therapeutic administration of chitin derivatives. Cell Mol Life Sci 1997; 53: 131-40.

9. Koga D, Chen R and Chen HC. Chitin enzymology-chitinase. In: Advances in Chitin Science. Elsevier Science, 1998; 16-23.

10. Chenite A, Chaput C, Wang D, et al. Novel injectable neutral solutions of chitosan form biodegradable gels in situ. Biomaterials 2000; 21: 2155-61.

11. Muzzarelli RA. Chitin and the human body. In: Advances in Chitin Science. Elsevier Science, Brest. 1995; 448-61.
12. Chatelet C, Damour O and Domard A. Influence of the degree of acetylation on some biological properties of chitosan films. Biomaterials 2001; 22: 261-8.

13. Felt O, Carrel A, Baehni P, et al. Chitosan as tear substitute: A wetting agent endowed with antimicrobial efficacy. J Ocul Pharmacol Ther 2000; 16: 261-70.

14. Liu XF, Guan YL, Yang DZ, et al. Antibacterial action of chitosan and carboxymethylated chitosan. J Appl Polym Sci 2001; 79: 1324-35.

15. Ouattara B, Simard RE, Piette G, et al. Inhibition of surface spoilage bacteria in processed meats by application of antimicrobial films prepared with chitosan. Int $J$ Food Microbiol 2000; 62: 139-48.

16. Peluso G, Petillo O, Ranieri M, et al. Chitosan-mediated stimulation of macrophage function. Biomaterials 1994; 15: 1215-20.

17. Vasudev SC, Chandy T and Sharma CP. Development of chitosan/polyethylene vinyl acetate co-matrix: Controlled release of aspirin-heparin for preventing cardiovascular thrombosis. Biomaterials 1997; 18: 375-81.

18. Muzzarelli RA, Biagini G, Bellardini M, et al. Osteoconduction exerted by methylpyrrolidione chitosan used in dental surgery. Biomaterials 1993; 14: 39-43.

19. Ikeda T, Yanagiguchi K, Viloria IL, et al. Relationship between lysozyme activity and clinical symptoms following the application of chitin/chitosan in endodontic treatment. In: Muzzarelli RA. ed. Chitosan per os: from dietary supplement to drug carrier, Atec, Grottammare. 2000; 275-92.

20. Muzzarelli RA, Biagini G and Belmonte M. Osteoinduction by chitosan-complexed BMP: Morpho-structural responses in an osteoporotic model. J Bioactive Com Polym 1997; 12: 321-9.

21. Seol YJ, Lee JY, Park YJ, et al. Chitosan sponges as tissue engineering scaffolds for bone formation. Biotechnol Lett 2004; 26: 1037-41.

22. Roth J and Warhol MJ. Immunogold silver staining techniques for high resolution immunohistochemistry in clinical materials. In: Bullock G, Van Velzen D and Warhol MJ. ed. Techniques in diagnostic pathology. vol 3. Academic Press, New York. 1992; 2-23.

23. Roth J. Application of lectin-gold complexes for electron microscopic localization of glycoconjugates on thin sections. $J$ Histochem Cytochem 1983; 31: 987-99.

24. Geoghegan WD and Ackerman GA. Adsorption of horseradish peroxidase, ovomucoid and anti-immunoglobulin to colloidal gold for the indirect detection of concanavalin A, wheat germ agglutinin and goat anti-human immunoglobulin $\mathrm{G}$ on cell surfaces at the electron microscopic level: A new method, theory and application. J Histochem Cytochem 1977; 25: 1187200.

25. Tachaboonyakiat W, Serizawa T and Akashi M. Inorganicorganic polymer hybrid scaffold for tissue engineering II: partial enzymatic degradation of hydroxyapatite-chitosan hybrid. J Biomater Sci Polym Ed 2002; 13: 1021-32.

26. Park JK, Shimono K, Ochiai N, et al. Purification, characterization, and gene analysis of a chitosanase (ChoA) from 
Matsuebacter chitosanotabidus 3001. J Bacteriol 1999; 181:

6642-9.

27. Cho YW, Jang J, Park CR, et al. Preparation and solubility in acid and water of partially deacetylated chitins. Biomacromolecules 2000; 1: 609-14.

28. Ruel-Gariepy E, Chenite A, Chaput C, et al. Characterization of thermosensitive chitosan gels for the sustained delivery of drugs. Int $J$ Pharm 2000; 203: 89-98.

29. Shim SY, Park HN, Kim KH, et al. Biological evaluation of chitosan nanofiber membrane for guided bone regeneration. J Periodontal 2005; 76: 1778-84.

30. Renbutsu E, Hirose M, Omura Y, et al. Preparation and biocompatibility of novel UV-curable chitosan derivatives. Biomacromolecules 2005; 6: 2385-8.

31. Gobin AS, Froude VE and Mathur AB. Structural and mechanical characteristics of silk fibroin and chitosan blend scaffolds for tissue regeneration. J Biomed Mater Res A 2005; 74: 465-73.

32. Chen MH, Chen RS, Hsu YH, et al. Proliferation and phenotypic preservation of rat parotid acinar cells. Tissue Eng 2005; 11: 526-34.

33. Ikeda T, Yanagiguchi K and Hayashi Y. Application to dental medicine. In focus on dental caries and alveolar bone healing (in Japanese). Bioindustry 2002; 19: 22-9.

34. Tomihata K. and Ikada Y. In vitro and in vivo degradation of films of chitin and its deacetylated derivatives. Biomaterials 1997; 18: 567-75.

35. Hidaka Y, Ito M, Mori K, et al. Histopathological and immunohistochemical studies of membranes of deacetylated chitin derivatives implanted over rat calvaria. J Biomed Mater Res 1999; 46: 418-23.

(Accepted for publication January 18, 2006) 